\title{
On the Efficient Simulation of the Distribution of the Sum of Gamma-Gamma Variates with Application to the Outage Probability Evaluation Over Fading Channels
}

\author{
Chaouki Ben Issaid, Nadhir Ben Rached, Abla Kammoun, Mohamed-Slim Alouini, and Raul Tempone
}

\begin{abstract}
The Gamma-Gamma distribution has recently emerged in a number of applications ranging from modeling scattering and reverberation in sonar and radar systems to modeling atmospheric turbulence in wireless optical channels. In this respect, assessing the outage probability achieved by some diversity techniques over this kind of channels is of major practical importance. In many circumstances, this is related to the difficult question of analyzing the statistics of a sum of GammaGamma random variables. Answering this question is not a simple matter. This is essentially because outage probabilities encountered in practice are often very small, and hence the use of classical Monte Carlo methods is not a reasonable choice. This lies behind the main motivation of the present work. In particular, this paper proposes a new approach to estimate the left tail of the sum of Gamma-Gamma variates. More specifically, we propose robust importance sampling schemes that efficiently evaluates the outage probability of diversity receivers over Gamma-Gamma fading channels. The proposed estimators satisfy the well-known bounded relative error criterion for both maximum ratio combining and equal gain combining cases. We show the accuracy and the efficiency of our approach compared to naive Monte Carlo via some selected numerical simulations.
\end{abstract}

Index Terms-Gamma-Gamma, generalized-K, importance sampling, Monte Carlo, bounded relative error, outage probability, maximum ratio combining.

\section{INTRODUCTION}

The Gamma-Gamma distribution has recently emerged in many communication systems. Not only that it generalizes other types of distributions that are used to model the statistics of the fading channels, such as the $\mathrm{K}$ and the double Rayleigh distribution for instance, but it also shows a good agreement with the measurements from the experiments conducted in $[1,2]$ for example. In radar and sonar systems, it has been used to model scattering [3] and reverbation [4]. It has been broadly accepted as an appropriate model for both line-of sight and non-line-of sight wireless radio-frequency channels $[5,6]$. Lately, it started to gain popularity in modeling atmospheric

The authors are in the Computer, Electrical and Mathematical Science and Engineering (CEMSE) Division, King Abdullah University of Science and Technology (KAUST), Thuwal, Makkah Province, Saudi Arabia. (e-mail: chaouki.benissaid, nadhir.benrached, abla.kammoun, slim.alouini, raul.tempone@kaust.edu.sa).

This work is in part supported by the Qatar National Research Fund (a member of Qatar Foundation) under NPRP Grant NPRP9-077-2-036 and in part by the KAUST-KFUPM initiative.

Part of this work was presented in the IEEE Global Conference on Signal and Information Processing (GlobalSIP'2016), Greater Washington, D.C., USA, Dec. 2016. turbulence in wireless optical channels [7, 8]. The GammaGamma distribution is a compound model since it presents the product of two Gamma random variables (RVs) that model the small and large scale fluctuations, respectively [9]. It was shown that this probability density function (PDF) models accurately the atmospheric turbulence in free-space optics (FSO) over a wide range being a good fit for both weak and strong turbulence regimes $[10,11]$. A concise review of the use of Gamma-Gamma to model the statistics of the signal fading channels can be found in [10].

The statistics of the sum of Gamma-Gamma RVs is needed, for instance, when investigating the efficiency of certain diversity techniques, e.g. maximum ratio combining (MRC) or equal gain combining (EGC) [12]. To the best of our knowledge, a closed-form expression for the distribution of the sum of independent and not necessarily identically distributed (i.n.i.d) Gamma-Gamma RVs, known also as the generalized$\mathrm{K}$ distribution, does not exist in the literature. In fact, the presence of the modified Bessel function of the second kind in the expression of the Gamma-Gamma PDF, makes this task analytically challenging [13]. In the independent and identically distributed (i.i.d) case, there have been few attempts to derive analytically the sum of Gamma-Gamma variates. The first attempt was in [14] where the authors presented the PDF of the sum in the case of i.i.d RVs as a nested series. The limitation of this derivation is that the fading parameters were assumed to be integers. For the non-integer case, an infinite series representation was derived in [15] using the generalized power series representation of the modified Bessel function of the second kind. The analytical complications and the computational burden of deriving the exact PDF of the sum led many authors to turn their attention to look for an approximate distribution. In [16], the authors used a single Gamma PDF as an approximate distribution of the generalized-K PDF. By matching the first moment and the amount of fading of the instantaneous power in both cases, the parameters of the Gamma PDF were derived for both the i.i.d and i.n.i.d scenarios. A similar approach was introduced in [17] where a Gamma-Gamma PDF is used to approximate the distribution of the sum. Matching the moment generating functions (MGFs) of the generalized-K and the Gamma-Gamma PDFs led to determine the expression of the parameters of the approximate PDF. In [13], the distribution of the sum was approximated by a single Gamma-Gamma 
distribution in the i.i.d case. In the i.n.i.d scenario, a nested finite weighted sum of Gamma-Gamma PDFs was used to fit the PDF of the sum. The latter approximation assumes that one of the shaping parameters, is the same for all variates. A more general distribution, $\alpha-\mu$, was used in [18], to fit the distribution of the sum in the i.i.d case. In his work, Peppas used the moment matching technique, and more specifically, the first, the second, and the fourth moments were used to determine the parameters of the $\alpha-\mu$ distribution. The sum of K-distributed RVs, a special case of the sum of GammaGamma variates, has been investigated in [19]. The authors have derived infinite series expressions for both the PDF and the cumulative distribution function (CDF) of the sum of K-distributed RVs. Capitalizing on these expressions, they have studied the system performance, in the presence of fading/shadowing, noise and interference, in terms of the average bit error probability and the outage probability. However, it is well-known that an approximation has the risk to lead to an inaccurate representation of the distribution of the sum of the $\mathrm{RVs}$ for a certain set of parameters. Indeed, although it often simplifies the analysis without producing large errors, it may be that such an approximation is sensitive to the parameters of the problem. Thus, it can be accurate for some values and not for others. For the sake of illustration, the authors in [17] have shown a clear discrepancy between simulation and the approximate approaches presented in $[13,16,18]$. To overcome this problem, one can resort to a numerical simulation approach, e.g. Monte Carlo (MC) method, which can provide more accurate results.

In this work, we are interested in estimating the probability of rare events (e.g. of the order of $10^{-8}$ ), for which the MC method is inaccurate if the sample size is not large enough [20]. In fact, a naive MC estimator, based on a reasonably limited number of simulation runs, may provide irrelevant results. For example, suppose we want to compute a probability $p=10^{-8}$ and we decided to use only $10^{6}$ samples. With a probability greater than 0.99 , the result will be equal to zero providing irrelevant information on the value of $p$ and the $99 \%$ confidence interval in this case is $\left[0,4.6 \times 10^{-6}\right]$, thus a grossly erroneous estimation [21]. If one wants to obtain reasonably accurate results when dealing with rare events, one can use variance reduction techniques, for instance the importance sampling (IS) method [22]. IS introduces a new distribution, often called biased PDF, that "encourages" the sampling of values from the importance region. The fundamental point in the implementation of a simulation using IS is the choice of the biased distribution. Constructing a good biased distribution is the art of IS. In fact, the advantage can then be a tremendous computational time savings while the disadvantage, in the case of a poor choice of biased distribution, is an estimator with a larger variance than a MC estimator. That is why, an IS estimator needs to satisfy certain criteria to guarantee the efficiency of the method.

In this paper, we propose efficient IS schemes for the estimation of the outage probability of multibranch MRC and EGC diversity receivers over Gamma-Gamma fading channels. More precisely, we select the biased PDF as a GammaGamma distribution with shifting a certain parameter in the functional form of the distribution. The main result of our work is that the proposed IS approaches possess the well-desired bounded relative error property, in both MRC and EGC cases, which means that the number of samples required to meet a fixed accuracy requirement remains bounded independently of how small is the outage probability. It is important to note that, to the best of the authors knowledge, the use of the IS method to estimate the outage probability with diversity techniques among researchers in wireless communications is quite limited. Recently, the authors in [23] have addressed this problem using two unified IS schemes. However, not only the i.i.d setting was assumed to show the bounded relative error property but also sampling according to their biased PDF is not straightforward in the Gamma-Gamma fading model scenario.

The reminder of this paper is organized as follows. We start by describing the system model in Section II. We then provide in Section III a brief description of the fundamental concepts of IS method. In section IV, we present our approach to estimate the outage probability in our particular set-up. We also discuss the efficiency of the proposed method in both the MRC and EGC cases. In Section V, we show some selected numerical simulations related to the outage probability of multibranch MRC and EGC diversity receivers over Gamma-Gamma fading channels. Finally, the paper ends with a summary of the main results.

Throughout this paper, we use the following notations: $\mathbb{P}(A)$ denotes the probability that the event $A$ will take place, $f_{X}(\cdot)$ (respectively $\left.f_{X}^{*}(\cdot)\right)$ is the original (respectively biased) PDF of the RV $X$. The notations $\mathbb{E}[Y], \mathbb{V}[Y]$ (respectively $\mathbb{E}^{*}[Y]$ and $\mathbb{V}^{*}[Y]$ ) stand respectively for the expectation and the variance of the random quantity $Y$ with respect to (w.r.t) the PDF $f_{X}(\cdot)$ (respectively $f_{X}^{*}(\cdot)$ ). We refer to the indicator function by $\mathbb{1}_{(\cdot)}$. We denote by $\Gamma \Gamma(k, m, \Omega)$ a Gamma-Gamma variate with parameters $(k, m, \Omega)$. Finally, for the limiting behavior of functions, $f=\mathcal{O}(g)$ means that $f$ is bounded above up to a constant factor by $g$ asymptotically and we use $f \sim g$ when $f$ is equal to $g$ asymptotically.

\section{SySTEM MOdEL}

The instantaneous signal-to-noise ratio (SNR) expression at the diversity receiver, is given by [24]

$$
\gamma_{\text {end }}=\frac{E_{s}}{N_{0} \sqrt{L^{1-p+q}}}\left(\sum_{\ell=1}^{L} X_{\ell}^{\frac{p}{2}}\right)^{q},
$$

where $(p, q)=(1,2)$ for the EGC case and $(p, q)=(2,1)$ for the MRC case. The ratio $\frac{E_{s}}{N_{0}}$ is the SNR per symbol at the transmitter, $L$ is the number of diversity branches. Eq. (1) is slightly modified compared to reference [24] in order to model the channel gains $\left\{X_{\ell}\right\}_{\ell=1}^{L}$ as i.n.i.d Gamma-Gamma variates $\Gamma \Gamma\left(k_{\ell}, m_{\ell}, \Omega_{\ell}\right), \ell=1, \ldots, L$, whose PDFs are given by [11]

$$
\begin{aligned}
f_{X_{\ell}}(x) & =\frac{2\left(k_{\ell} m_{\ell}\right)^{\frac{k_{\ell}+m_{\ell}}{2}} x^{\frac{k_{\ell}+m_{\ell}}{2}-1}}{\Gamma\left(m_{\ell}\right) \Gamma\left(k_{\ell}\right) \Omega_{\ell}^{\frac{k_{\ell}+m_{\ell}}{2}}} K_{k_{\ell}-m_{\ell}}\left(2\left(\frac{k_{\ell} m_{\ell}}{\Omega_{\ell}} x\right)^{\frac{1}{2}}\right), \\
x & \geq 0, \ell=1, \ldots, L
\end{aligned}
$$

where $k_{\ell}$ and $m_{\ell}$ are two positive real numbers that represent the distribution parameters, $K_{\nu}(\cdot)$ is the modified Bessel 
function of the second kind of order $\nu$ [25, Sec. (8.432)], $\Gamma(\cdot)$ is the Gamma function [25, Sec. (8.31)], and $\Omega_{\ell}$ is the mean power of the RV $X_{\ell}, \ell=1, \ldots, L$.

The Gamma-Gamma distribution $\Gamma \Gamma(k, m, \Omega)$ generalizes other types of PDFs when certain values of the fading parameters are considered. For instance, it is a good approximation for the Gamma distribution when $k \rightarrow+\infty$ or $m \rightarrow+\infty$. For $m=1$ or $k=1$, it coincides with a K-distribution, while when $k=1$ and $m=1$, it reduces to the square of the double Rayleigh distribution. In small perturbations, the Gamma-Gamma model gives similar results to the log-normal model [11] and fits the simulation data performed by Flatté et al. [26]. Also, since the K-distribution is a special case of the Gamma-Gamma distribution, it can also model strong turbulence regime. To sum up, the Gamma-Gamma turbulence model allows to describe different turbulence regimes and shows a good fit with data from measurements [11].

The outage probability $P$, which quantifies the probability that the instantaneous SNR falls below a certain threshold $\gamma_{t h}$, is frequently used as a performance metric of communication systems operating over fading channels

$P=\mathbb{P}\left(\gamma_{\text {end }} \leq \gamma_{t h}\right)=\mathbb{P}\left(\sum_{\ell=1}^{L} X_{\ell}^{\frac{p}{2}} \leq\left(\frac{N_{0}}{E_{s}} \sqrt{L^{1-p+q}} \gamma_{t h}\right)^{\frac{1}{2}}\right)$

At a higher level of abstraction, our aim is to find the CDF of the sum of Gamma-Gamma RVs. More specifically, we are interested in the case in which the outage probability requirements are very low, i.e. of the order $10^{-8}$. This situation occurs, for instance, when studying the performance of FSO systems since they are often used for high-speed backhaul wireless links which aggregate the data generated by multiple users [27].

\section{IMPORTANCE SAMPLING}

As stated previously, no closed-form results for the CDF of the sum of i.n.i.d Gamma-Gamma variates were derived in the literature. For the reader convenience, we recall first the main concepts behind IS. This will facilitate the understanding of the proposed approach that will be discussed in depth later.

Writing $P=\mathbb{E}\left[\mathbb{1}_{\left(S_{L} \leq \gamma_{0}\right)}\right]$, the naive MC estimator of (3) is given by

$$
\hat{P}_{M C}=\frac{1}{N} \sum_{i=1}^{N} \mathbb{1}_{\left(S_{L}\left(\omega_{i}\right) \leq \gamma_{0}\right)},
$$

where $N$ is the number of MC samples, and $\left\{S_{L}\left(\omega_{i}\right)\right\}_{i=1}^{N}$ are i.i.d. realizations of the RV $S_{L}$. The sequence $\left\{X_{\ell}\left(\omega_{i}\right)\right\}_{\ell=1}^{L}$ is sampled independently according to the PDFs (2), for each realization of $S_{L}$.

When the value of $P$ is very small, naive MC turns out to be computationally expensive. In fact, in this setting, a very large number of samples $N$, of the order of $100 / P$, is required to ensure that $\hat{P}_{M C}$ estimates accurately the quantity of interest with $10 \%$ relative error.

An alternative method to evaluate the probability of rare events is the IS technique [22]. In addition to reducing the computational work compared to naive MC, IS is known for its simplicity and ease of implementation compared to the other variance reduction techniques. The main idea behind this method is to construct an unbiased estimator of $P$, with smaller variance than the naive $\mathrm{MC}$ estimator, by introducing an auxiliary PDF $f_{X_{\ell}}^{*}(\cdot)$. For a concise review of the use of IS in communication systems, the reader is referred to [28].

IS exploits the fact that the representation of $P$ as an expected value is not unique. In fact, we can re-write $P$ as

$$
\begin{aligned}
P & =\mathbb{E}\left[\mathbb{1}_{\left(S_{L} \leq \gamma_{0}\right)}\right]=\int_{\mathbb{R}^{L}} \mathbb{1}_{\left(S_{L} \leq \gamma_{0}\right)} \prod_{\ell=1}^{L} f_{X_{\ell}}\left(x_{\ell}\right) d x_{1} \ldots d x_{L} \\
& =\int_{\mathbb{R}^{L}} \mathbb{1}_{\left(S_{L} \leq \gamma_{0}\right)} \mathcal{L}\left(x_{1}, \ldots, x_{L}\right) \prod_{\ell=1}^{L} f_{X_{\ell}}^{*}\left(x_{\ell}\right) d x_{1} \ldots d x_{L} \\
& =\mathbb{E}^{*}\left[\mathbb{1}_{\left(S_{L} \leq \gamma_{0}\right)} \mathcal{L}\left(X_{1}, \ldots, X_{L}\right)\right] .
\end{aligned}
$$

The likelihood ratio is defined as

$$
\mathcal{L}\left(X_{1}, \ldots, X_{L}\right)=\prod_{\ell=1}^{L} \frac{f_{X_{\ell}}\left(X_{\ell}\right)}{f_{X_{\ell}}^{*}\left(X_{\ell}\right)} .
$$

By defining the biased densities $\left\{f_{X_{\ell}}^{*}(\cdot)\right\}_{\ell=1}^{L}$, IS aims to "encourage" the sampling from the importance region $\left\{S_{L} \leq \gamma_{0}\right\}$. In this case, the IS estimator of (3) is

$$
\hat{P}_{I S}=\frac{1}{N^{*}} \sum_{i=1}^{N^{*}} \mathbb{1}_{\left(S_{L}\left(\omega_{i}\right) \leq \gamma_{0}\right)} \mathcal{L}\left(X_{1}\left(\omega_{i}\right), \ldots, X_{L}\left(\omega_{i}\right)\right),
$$

where for each realization $i=1, \ldots, N$, the sequence $\left\{X_{\ell}\left(\omega_{i}\right)\right\}_{\ell=1}^{L}$ are sampled independently according to the biased PDFs $\left\{f_{\ell}^{*}(\cdot)\right\}_{\ell=1}^{L}$. The use of a biased distribution will lead to a biased estimator if we apply it directly to the simulations. However, the different simulations are weighted in order to correct this bias, thereby the IS estimator is unbiased. The weight that is given to each simulation is the likelihood ratio which is the Radon-Nikodym density of the original distribution w.r.t the biased one. The main difficulty with the IS implementation is the right choice of the biased PDFs $\left\{f_{X_{\ell}}^{*}(\cdot)\right\}_{\ell=1}^{L}$. A bad choice can produce a large likelihood ratio. In order to avoid such a situation, many criteria have been used in the literature in order to characterize the goodness of an IS approach [29] among which we mention the bounded relative error. This latter represents one of the desirable property in the field of rare events algorithms.

Definition 1. The IS estimator has a bounded relative error if the following statement holds

$$
\limsup _{\gamma_{0} \rightarrow 0} \frac{\mathbb{E}^{*}\left[\mathbb{1}_{\left(S_{L} \leq \gamma_{0}\right)} \mathcal{L}^{2}\left(X_{1}, \ldots, X_{L}\right)\right]}{P^{2}}<+\infty .
$$

This criterion can be seen as a measure of robustness of the IS estimator. In fact, if it holds, then the number of simulation runs $N$ needed to achieve a fixed accuracy requirement remains bounded independently of how small the outage probability $P$ is. This has to be compared to naive MC simulation which requires the number of samples to grow as $\mathcal{O}\left(P^{-1}\right)$ in order to retrieve the same accuracy.

To quantify the efficiency of IS compared to naive MC, we introduce the following two metrics 
Definition 2. The relative error of naive MC simulation is given by

$$
\varepsilon=\frac{C}{P} \sqrt{\frac{P(1-P)}{N}}
$$

where $C=1.96$ which corresponds to a $95 \%$ confidence interval. From the above definition, we can see that the relative error is nothing but the ratio of half-width of the confidence interval over the estimated value.

Similarly, we define the relative error of an IS approach as

$$
\varepsilon^{*}=\frac{C}{P} \sqrt{\frac{\mathbb{V} *\left[\mathbb{1}_{\left(S_{L} \leq \gamma_{0}\right)} \mathcal{L}\left(X_{1}, \ldots, X_{L}\right)\right]}{N}} .
$$

Definition 3. If we fix the relative error requirement, then, we can easily determine the number of required simulation runs. In fact, for a fixed $\epsilon_{0}$ and using Eqs. (9) and (10), the number of samples needed by naive MC simulations and IS are respectively given by

$$
\begin{aligned}
N & =(P(1-P))\left(\frac{C}{P \epsilon_{0}}\right)^{2}, \\
N^{*} & =\mathbb{V}^{*}\left[\mathbb{1}_{\left(S_{L} \leq \gamma_{0}\right)} \mathcal{L}\left(X_{1}, \ldots, X_{L}\right)\right]\left(\frac{C}{P \epsilon_{0}}\right)^{2} .
\end{aligned}
$$

In the following section, we present a clever choice of the biased PDF in both MRC and EGC cases. Moreover, the efficiency of our proposed IS approach is studied for both scenarios.

\section{Proposed Approach}

\section{A. MRC case}

The outage probability in the MRC case is given by

$$
P=\mathbb{P}\left(\gamma_{\text {end }} \leq \gamma_{\text {th }}\right)=\mathbb{P}\left(S_{L} \leq \gamma_{0}\right),
$$

where $S_{L}=\sum_{\ell=1}^{L} X_{\ell}$ and $\gamma_{0}=\frac{N_{0}}{E_{s}} \gamma_{t h}$.

In this subsection, we propose to shift the mean of each variate under the original PDF, i.e. the mean under the biased PDF of $X_{\ell}$ is $\Omega_{\ell}^{*}=\Omega_{\ell}-\Omega_{0, \ell}$ where $\Omega_{0, \ell}$ satisfies $0 \leq \Omega_{0, \ell}<\Omega_{\ell}$ and as $\gamma_{0} \rightarrow 0$, it approaches $\Omega_{\ell}, \ell=1, \ldots, L$. In this case, the biased PDF is

$$
\begin{aligned}
& f_{X_{\ell}}^{*}\left(X_{\ell}\right)=\frac{2\left(k_{\ell} m_{\ell}\right)^{\frac{k_{\ell}+m_{\ell}}{2}} X_{\ell}^{\frac{k_{\ell}+m_{\ell}}{2}-1}}{\Gamma\left(m_{\ell}\right) \Gamma\left(k_{\ell}\right)\left(\Omega_{\ell}-\Omega_{0, \ell}\right)^{\frac{k_{\ell}+m_{\ell}}{2}}} \\
& \times K_{k_{\ell}-m_{\ell}}\left(2\left(\frac{k_{\ell} m_{\ell}}{\Omega_{\ell}-\Omega_{0, \ell}} X_{\ell}\right)^{\frac{1}{2}}\right), x \geq 0, \ell=1, \ldots, L .
\end{aligned}
$$

Inspired by the i.i.d case, we select $\Omega_{0, \ell}$ to be as follows

$$
\Omega_{0, \ell}=\Omega_{\ell}-\frac{\gamma_{0}}{L}, \forall \ell=1, \ldots L .
$$

Our choice of this particular biased PDF (14) is mainly motivated by the fact that, as we decrease the threshold and hence we decrease the outage probability, $\Omega^{*}$ will approach zero and thus samples from the region of interest $\left\{S_{L} \leq \gamma_{0}\right\}$ will take place more frequently. To validate the previous statement, we plot in Fig. 1 the PDF of $S_{2}=X_{1}+X_{2}$

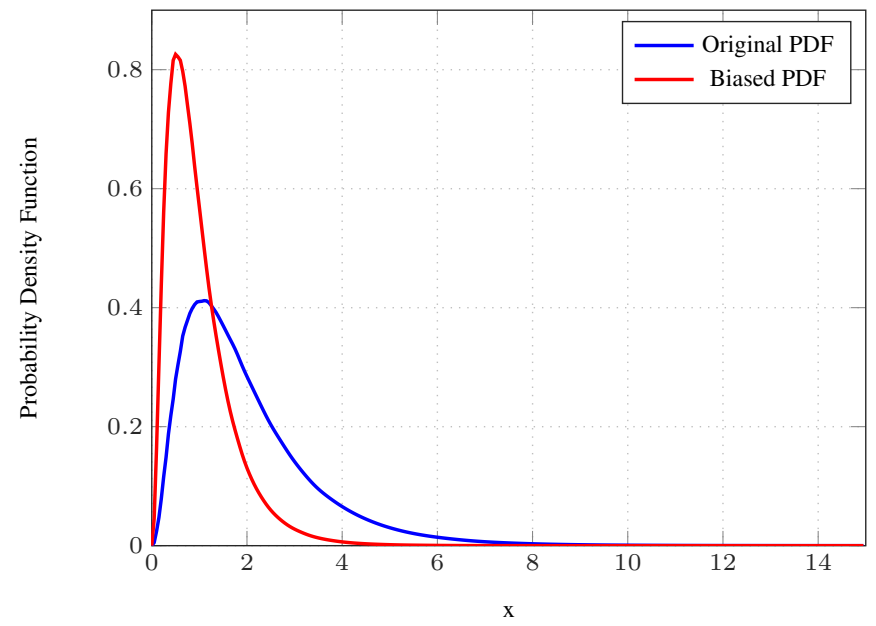

Fig. 1. PDF of the sum of two i.i.d Gamma-Gamma RVs with $k=3.99$, $m=1.7, \Omega=1$, and $\Omega_{0}=0.5$.

under the original and the biased distributions in the i.i.d setting. From this figure, we observe that the distribution of $S_{2}$ when $X_{1}$ and $X_{2}$ are sampled from the biased PDFs is more concentrated to the left tail than when they are sampled from their original PDFs. Thus, important samples, i.e. samples that belong to $\left\{S_{2}<\gamma_{0}\right\}$, are more likely to occur when sampling according to the biased PDFs than from the original ones.

Remark 1. Note that the sequence of parameters $\left\{\Omega_{0, \ell}\right\}_{\ell=1}^{L}$ defined in (15) represents a particular solution of the equation $\mathbb{E}^{*}\left[\sum_{\ell=1}^{L} X_{\ell}\right]=\gamma_{0}$.

With the value of $\Omega_{0}$ at hand, we characterize in the following theorem the robustness of the proposed IS approach. In fact, we show that it achieves the bounded relative error property which represents one of the most relevant criterion measuring the efficiency of IS schemes.

Theorem 1. Let $\left\{X_{\ell}\right\}_{\ell=1}^{L}$ be a sequence of i.n.i.d GammaGamma RVs and $f_{X_{\ell}}^{*}(\cdot)$ be defined as in (14) where $\Omega_{0, \ell}$ is given by (15). Then, the IS estimator (7) has a bounded relative error provided that $\min _{1 \leq \ell \leq L}\left(k_{\ell}-m_{\ell}\right)>\frac{1}{2}$ and $k_{\ell}-m_{\ell} \notin \mathbb{N}$, $\ell=1, \ldots, L$.

Proof: See Appendix A.

\section{B. EGC case}

For the EGC case, the outage probability expression is defined as

$$
P=\mathbb{P}\left(\gamma_{\text {end }} \leq \gamma_{t h}\right)=\mathbb{P}\left(T_{L} \leq \eta_{0}\right),
$$

where $T_{L}=\sum_{\ell=1}^{L} \sqrt{X_{\ell}}$ and $\eta_{0}=\left(\frac{N_{0} L}{E_{s}} \gamma_{t h}\right)^{\frac{1}{2}}$.

The PDF of $Z_{\ell}=\sqrt{X_{\ell}}$ is given by

$f_{Z_{\ell}}(z)=\frac{4\left(k_{\ell} m_{\ell}\right)^{\frac{k_{\ell}+m_{\ell}}{2}} z^{k_{\ell}+m_{\ell}-1}}{\Gamma\left(m_{\ell}\right) \Gamma\left(k_{\ell}\right) \Omega_{\ell}^{\frac{k_{\ell}+m_{\ell}}{2}}} K_{k_{\ell}-m_{\ell}}\left(2\left(\frac{k_{\ell} m_{\ell}}{\Omega_{\ell}}\right)^{\frac{1}{2}} z\right)$. 
Using the same kind of transformation as in the MRC case, the biased PDF can be written as

$$
\begin{aligned}
f_{Z_{\ell}}^{*}(z) & =\frac{4\left(k_{\ell} m_{\ell}\right)^{\frac{k_{\ell}+m_{\ell}}{2}} z^{k_{\ell}+m_{\ell}-1}}{\Gamma\left(m_{\ell}\right) \Gamma\left(k_{\ell}\right)\left(\Omega_{\ell}-\Omega_{0, \ell}\right)^{\frac{k_{\ell}+m_{\ell}}{2}}} \\
& \times K_{k_{\ell}-m_{\ell}}\left(2\left(\frac{k_{\ell} m_{\ell}}{\Omega_{\ell}-\Omega_{0, \ell}}\right)^{\frac{1}{2}} z\right) .
\end{aligned}
$$

Under the biased PDF, the mean of the RV $Z_{\ell}$ is given by

$$
\mathbb{E}^{*}\left[Z_{\ell}\right]=\frac{\Gamma\left(m_{\ell}+\frac{1}{2}\right) \Gamma\left(k_{\ell}+\frac{1}{2}\right)}{\Gamma\left(m_{\ell}\right) \Gamma\left(k_{\ell}\right)}\left(\frac{\Omega_{\ell}-\Omega_{0, \ell}}{k_{\ell} m_{\ell}}\right)^{\frac{1}{2}} .
$$

Regarding the choice of the parameter $\Omega_{0, \ell}$, we follow a similar approach to the MRC case. A particular solution of the equation $\mathbb{E}^{*}\left[\sum_{\ell=1}^{L} Z_{\ell}\right]=\eta_{0}$ is chosen

$$
\Omega_{0, \ell}=\Omega_{\ell}-\frac{\alpha_{\ell}}{L^{2}} \eta_{0}^{2}
$$

where $\alpha_{\ell}=k_{\ell} m_{\ell}\left[\frac{\Gamma\left(m_{\ell}\right) \Gamma\left(k_{\ell}\right)}{\Gamma\left(m_{\ell}+\frac{1}{2}\right) \Gamma\left(k_{\ell}+\frac{1}{2}\right)}\right]^{2}$.

Theorem 2. Let $\left\{Z_{\ell}\right\}_{\ell=1}^{L}$ be i.n.i.d square root of GammaGamma variates and $f_{Z_{\ell}}^{*}(\cdot)$ be defined as in (18) where $\Omega_{0, \ell}$ is given by (20). Thus, the IS estimator (7) has a bounded relative error when the conditions $\min _{1 \leq \ell \leq L}\left(k_{\ell}-m_{\ell}\right)>\frac{1}{2}$ and $k_{\ell}-m_{\ell} \notin \mathbb{N}, \ell=1, \ldots, L$ hold.

\section{Proof: See Appendix B.}

Remark 2. The variance of $Z_{\ell}$ under the new PDF is given by

$$
\mathbb{V}^{*}\left[Z_{\ell}\right]=\left[1-\frac{\Gamma\left(m_{\ell}+\frac{1}{2}\right) \Gamma\left(k_{\ell}+\frac{1}{2}\right)}{k_{\ell} m_{\ell} \Gamma\left(m_{\ell}\right) \Gamma\left(k_{\ell}\right)}\right]\left(\Omega_{\ell}-\Omega_{0, \ell}\right) .
$$

From the above expression, we can see that the choice of $\Omega_{0, \ell}$ in (20) will lead to reducing the variance as $\eta_{0} \rightarrow 0$.

Remark 3. Theorem 1. can be extended to the case when $\max _{1 \leq \ell \leq L}\left(k_{\ell}-m_{\ell}\right)<-\frac{1}{2}$.

Using that for $x \geq 0, \nu \mapsto K_{\nu}(x)$ is even, we can write $K_{k_{\ell}-m_{\ell}}(x)=K_{m_{\ell}-k_{\ell}}(x)$, where $m_{\ell}-k_{\ell}>\frac{1}{2}$. Then, we can use the bound [30, Eq.(1.3)] for $\nu=m_{\ell}-k_{\ell}$. Note also that since in this case $m_{\ell}>k_{\ell}$, then the asymptotic expansion of the CDF of $X_{\ell}$ around $x=0$ is given by

$$
\begin{gathered}
F_{X_{\ell}}(x) \underset{x \rightarrow 0}{\sim} \frac{\Gamma\left(m_{\ell}-k_{\ell}\right)}{\Gamma\left(m_{\ell}\right) \Gamma\left(k_{\ell}+1\right)}\left(\frac{k_{\ell} m_{\ell}}{\Omega_{\ell}} x\right)^{k}, m_{\ell}>k_{\ell}, \\
m_{\ell}-k_{\ell} \notin \mathbb{N} .
\end{gathered}
$$

Thereby, we can prove that the proposed IS estimator still has the bounded relative error property. Similar argument can be used to extend Theorem 2.

Remark 4. It is worth mentioning that in FSO, the fading parameters $k$ and $m$ are related to Rytov variance $\sigma_{R}^{2}$. Depending on the value of this variance, we can characterize the severity of the atmospheric turbulence. In fact, according to [31], a Rytov variance less than 0.3 corresponds to a weak turbulence, while moderate to strong turbulence is characterized by a value greater than 0.3 .

For a plane wave propagation, the expression of $k$ and $m$ are given by [11]

$$
\begin{aligned}
& k=\left[\exp \left(\frac{0.49 \sigma_{R}^{2}}{\left(1+1.11 \sigma_{R}^{\frac{12}{5}}\right)^{\frac{7}{6}}}\right)-1\right]^{-1}, \\
& m=\left[\exp \left(\frac{0.51 \sigma_{R}^{2}}{\left(1+0.69 \sigma_{R}^{\frac{12}{5}}\right)^{\frac{5}{6}}}\right)-1\right]^{-1} .
\end{aligned}
$$

In Fig. 2, we plot the difference $k-m$ and we observe that such a difference is always bigger than $\frac{1}{2}$. In fact, we can show that in this case $k-m \geq \frac{3}{2}$. Also, we can easily observe that $k-m \notin \mathbb{N}$. Therefore, the conditions $k-m>\frac{1}{2}$ and $k-m \notin \mathbb{N}$ can be seen as reasonable assumptions when studying the performance of FSO systems using model (23).

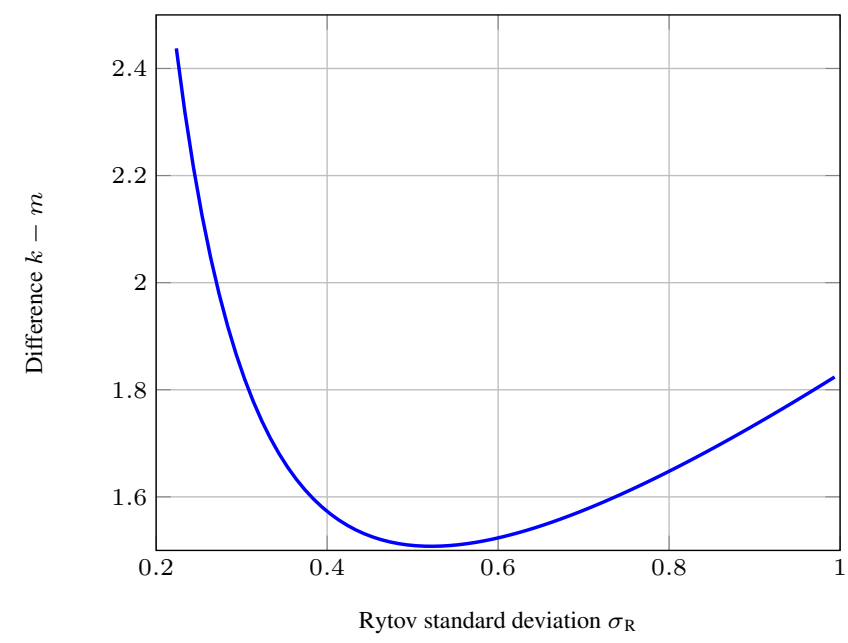

Fig. 2. Difference $k-m$ as function of Rytov standard deviation $\sigma_{R}$ assuming model (23).

\section{Simulation Results}

In this section, the outage probability is estimated using both the naive MC as well as the proposed IS approach described in Section IV. First, the accuracy of these two methods is analyzed in both MRC and EGC settings. Then, the gain, in terms of required number of samples needed to meet a fixed accuracy requirement, achieved by IS w.r.t naive MC is quantified.

\section{A. MRC Case}

We consider the estimation of the outage probability of L-branch MRC diversity receivers in the case of GammaGamma fading model. The set of parameters of this experiment are given in Table I. For the computation of the parameters $\left(k_{\ell}, m_{\ell}\right)$, we use the model given by (23) for different Rytov variance values. For three different choices of $L \in\{4,5,6\}$, we plot in Fig. 3 the outage probability estimated by both standard MC and our IS approach as a function of the SNR threshold $\gamma_{t h}$. Although there is a good agreement between 
MC and IS for high values of outage probabilities for each value of $L$, naive MC, unlike IS, fails to estimate the low outage probabilities. For instance, for $L=4$, the naive MC estimator matches perfectly the IS estimator up to an outage probability value of the order of $10^{-5}$. Then, as we decrease the SNR threshold, the MC estimate becomes erroneous. While only $10^{4}$ simulation runs are sufficient for IS to yield a very accurate estimate of the outage probability, much more than $10^{7}$ samples must be used by naive MC in order to retrieve the same accuracy.

TABLE I

FADING PARAMETERS USED TO SIMULATE THE OUTAGE PROBABILITY OF L-BRANCH DIVERSITY RECEIVERS OVER I.N.I.D GAMMA-GAMMA FADING MODEL IN FIG. 3.

\begin{tabular}{c|c}
\hline$L$ & Fading Parameters $\left(k_{\ell}, m_{\ell}\right)$ \\
\hline \hline 4 & $(3.99,1.7),(5.41,3.78),(4.05,1.98),(8.43,6.92)$ \\
\hline 5 & $(3.99,1.7),(5.41,3.78),(4.05,1.98),(4.39,2.56),(8.43,6.92)$ \\
\hline 6 & $(3.99,1.7),(5.41,3.78),(4.05,1.98)$ \\
& $(4.39,2.56),(4.74,3.01),(8.43,6.92)$ \\
\hline
\end{tabular}

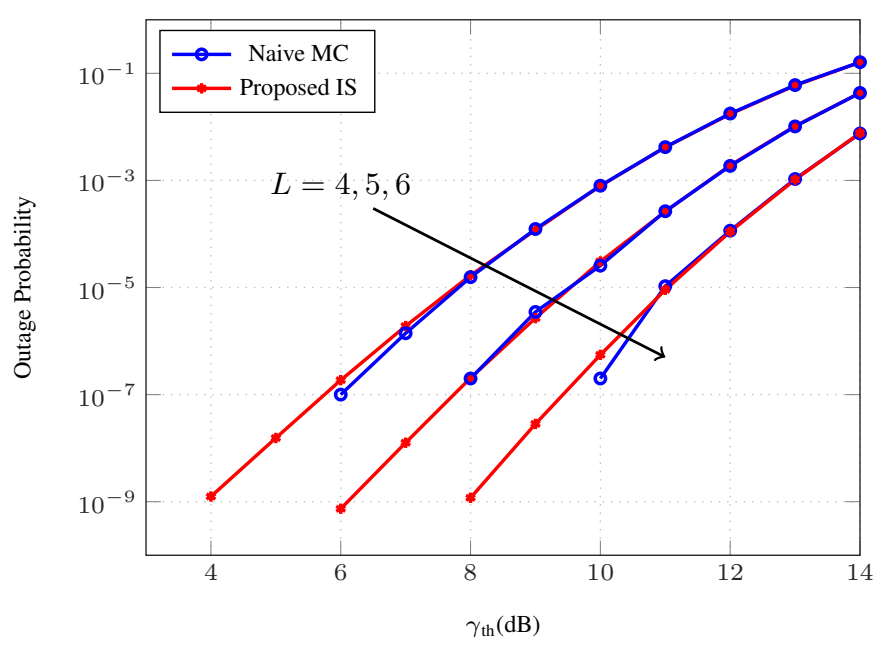

Fig. 3. Outage probability of L-branch MRC diversity receivers over GammaGamma fading model with $E_{s} / N_{0}=10 \mathrm{~dB}, \Omega_{\ell}=0 \mathrm{~dB}, \ell=1, \ldots, L$, and fading parameters of Table I. Number of samples $N=10^{7}$ and $N^{*}=10^{4}$.

The computational efficiency is investigated in Fig. 4. To guarantee a $95 \%$ accuracy level, $N^{*}$ remains almost constant independently of how small the outage probability is. This fact is expected due to the bounded relative error criterion. On the other hand, as we decrease the SNR threshold, we observe that $N$ grows with a very high rate. For instance, for $L=4$ and $\gamma_{t h}=4 \mathrm{~dB}$, approximately $1.2 \times 10^{12}$ samples are required by naive $\mathrm{MC}$ simulations to ensure a $95 \%$ accuracy requirement, whereas only $3.1 \times 10^{4}$ simulation runs are sufficient for the proposed IS approach to meet the same accuracy. This goes in favor toward the high computational out-performance of the newly proposed IS scheme w.r.t naive MC simulations.

In Fig. 5, we plot the relative errors of both methods for the case $L=4$. For large outage probability values, the relative error of naive MC is slightly smaller than IS. However, we observe that as the outage probability becomes smaller, the relative error of IS remains almost constant, in agreement with the bounded relative error property, while the naive MC

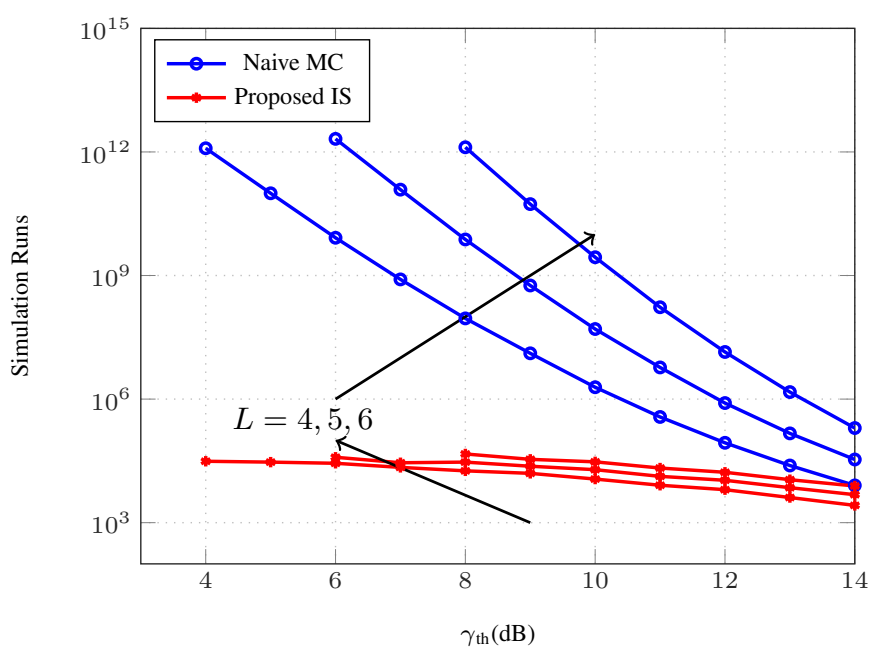

Fig. 4. Number of required simulation runs for $5 \%$ relative error for L-branch MRC diversity receivers over Gamma-Gamma fading model with $E_{s} / N_{0}=$ $10 \mathrm{~dB}, \Omega_{\ell}=0 \mathrm{~dB}, \ell=1, \ldots, L$, and fading parameters of Table I.

relative error grows rapidly although the number of samples used for MC is $10^{4}$ times greater than the one used for IS simulation. This observation highlights again the efficiency of the proposed IS estimator compared to naive MC when dealing with small outage probabilities.

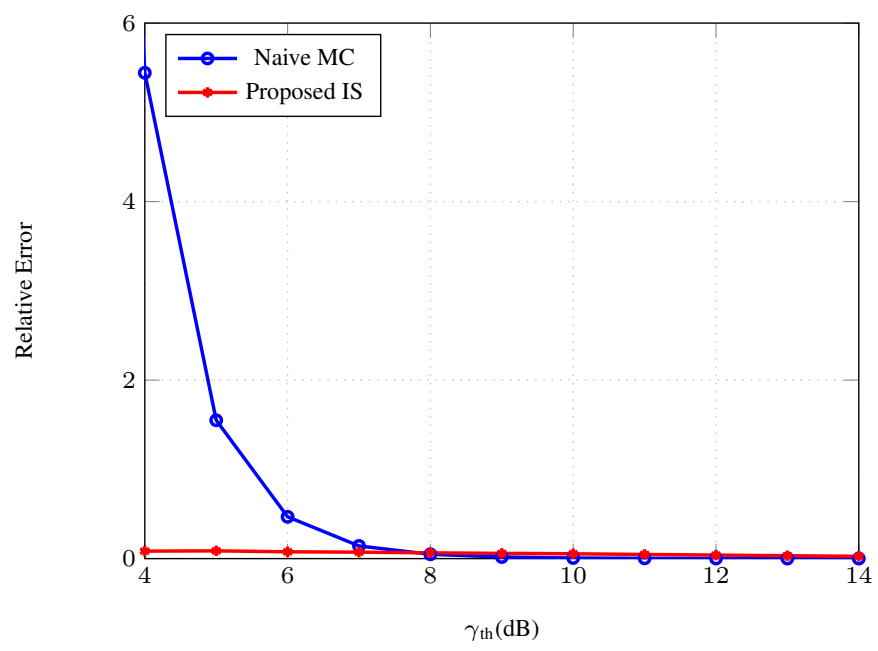

Fig. 5. Relative error of both methods for $L=4$ with number of samples $N=10^{8}$ and $N^{*}=10^{4}$.

\section{B. EGC case}

Now, we turn our attention to study the outage probability of $L$-branch EGC diversity receivers. The simulation parameters are the same as in Table I. The behavior of the outage probability as a function of the SNR threshold $\gamma_{t h}$, is depicted in Fig. 6 for three different values of the number of branches $L=4,5$, and 6 . The number of simulation runs used here is $N=10^{7}$ for MC and $N^{*}=10^{4}$ for IS. Similar conclusions are drawn as in the previous subsection. In fact, with few number of simulation runs, our IS scheme provides again a highly accurate estimate compared to the standard MC approach. 


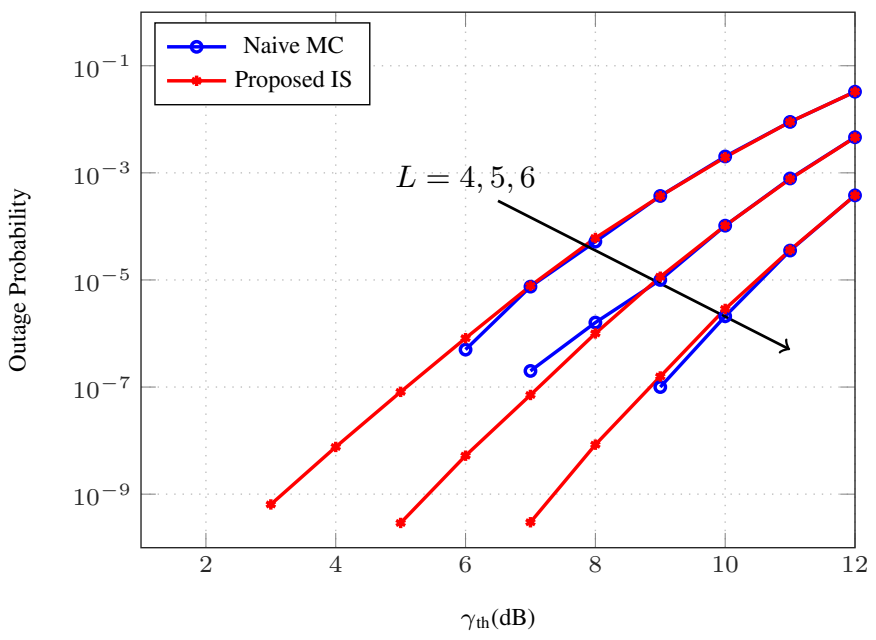

Fig. 6. Outage probability of L-branch EGC diversity receivers over GammaGamma fading model with $E_{s} / N_{0}=10 \mathrm{~dB}, \Omega_{\ell}=0 \mathrm{~dB}, \ell=1, \ldots, L$, and fading parameters of Table I. Number of samples $N=10^{7}$ and $N^{*}=10^{4}$.

To have a clear idea about the efficiency of our proposed IS estimator, we turn our attention to Fig. 7. To this end, we set both relative errors given by Definition 2 to be $\varepsilon=\varepsilon^{*}=5 \%$, and we compute, using the expressions detailed in Definition 3, the number of simulation runs $N$ and $N^{*}$ needed respectively by naive MC and IS to meet the aforementioned $95 \%$ accuracy level. From this figure, it is clearly obvious that the proposed IS method outperforms naive MC simulation in all of the three considered scenarios. Furthermore, we note that as the SNR threshold decreases, the efficiency increases. In fact, for each scenario, the number of samples $N$ is rapidly growing as the outage probability becomes smaller, whereas $N^{*}$ is almost constant over the considered range of outage probabilities. The latter statement is a consequence of the bounded relative error property of the IS estimator. For the sake of illustration, for $L=4$, the number of samples $N^{*}$ required by IS is approximately $7 \times 10^{4}$ (respectively $9 \times 10^{7}$ ) times less than the number of samples used in MC simulations for $\gamma_{t h}=4$ $\mathrm{dB}$ (respectively $\gamma_{t h}=1 \mathrm{~dB}$ ).

In Fig. 8, we plot the relative errors of both methods for the case $L=4$. Similar conclusions to the MRC case can also be drawn in this setting. In fact, it is clear that the variation of the relative error of the IS is much slower than that of the standard MC.

\section{CONCLUSION}

In this paper, we proposed efficient IS schemes for the estimation of the left tail of the sum of Gamma-Gamma variates as well as the sum of the square root of Gamma-Gamma variates. These schemes were used to efficiently estimate the outage probability of multibranch MRC and EGC diversity receivers over Gamma-Gamma fading channels. We showed that the proposed estimators possess the bounded relative error for both MRC and EGC cases. Simulation results show a significant reduction in the number of samples for the same level of accuracy which highlights the efficiency of the proposed IS estimator compared to naive MC.

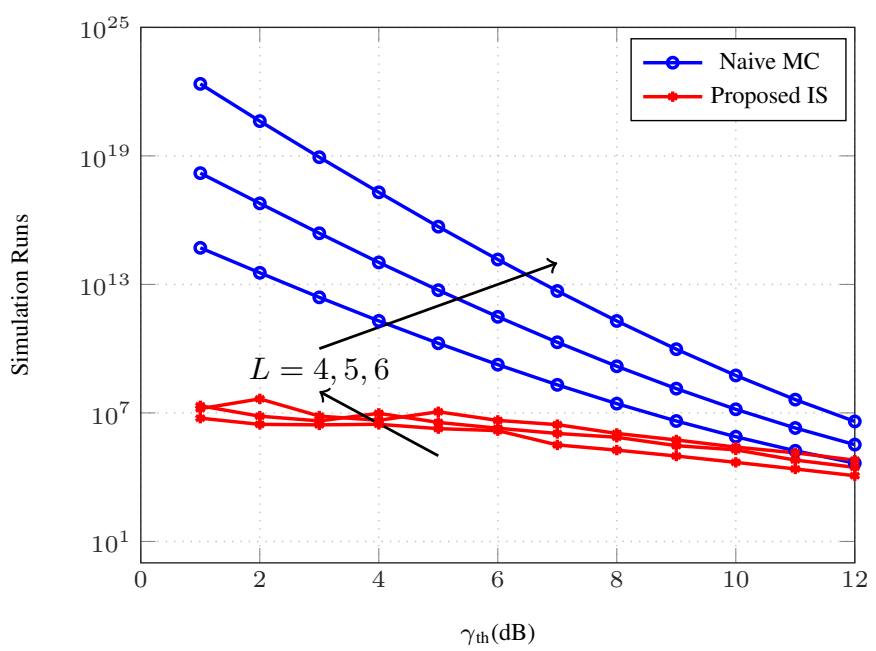

Fig. 7. Number of required simulation runs for $5 \%$ relative error for L-branch EGC diversity receivers over Gamma-Gamma fading model with $E_{s} / N_{0}=$ $10 \mathrm{~dB}, \Omega_{\ell}=0 \mathrm{~dB}, \ell=1, \ldots, L$, and fading parameters of Table I.

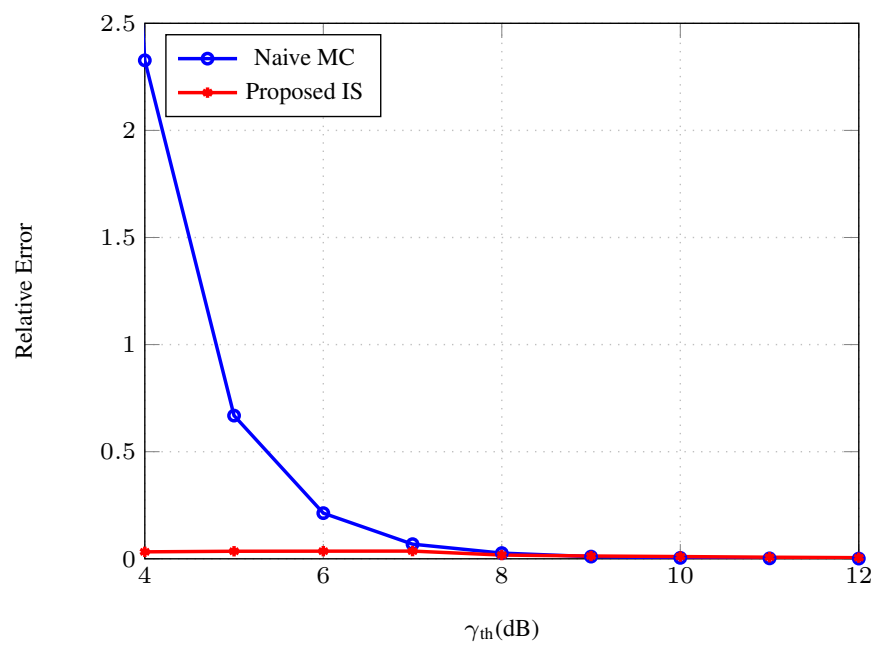

Fig. 8. Relative error of both methods for $L=4$ with number of samples $N=10^{8}$ and $N^{*}=10^{4}$.

\section{APPENDIX A}

ProOF OF THEOREM 1

Proof: To prove the Theorem 1, we recall first the definition of the likelihood ratio

$$
\begin{aligned}
& \mathcal{L}\left(X_{1}, \ldots, X_{L}\right)=\prod_{\ell=1}^{L} \frac{f_{X_{\ell}}\left(X_{\ell}\right)}{f_{X_{\ell}}^{*}\left(X_{\ell}\right)} \\
& =\prod_{\ell=1}^{L}\left(\frac{\Omega_{\ell}-\Omega_{0, \ell}}{\Omega_{\ell}}\right)^{\frac{L}{2}\left(k_{\ell}+m_{\ell}\right)} \frac{K_{k_{\ell}-m_{\ell}}\left(2\left(\frac{k_{\ell} m_{\ell}}{\Omega_{\ell}} X_{\ell}\right)^{\frac{1}{2}}\right)}{K_{k_{\ell}-m_{\ell}}\left(2\left(\frac{k_{\ell} m_{\ell}}{\Omega_{\ell}-\Omega_{0, \ell}} X_{\ell}\right)^{\frac{1}{2}}\right)} .
\end{aligned}
$$

We bound the ratio of the modified Bessel function of the second kind, in the case when $\min _{1 \leq \ell \leq L}\left(k_{\ell}-m_{\ell}\right)>\frac{1}{2}$, using 
[30, Eq.(1.3)]

$$
\begin{aligned}
& \frac{K_{k_{\ell}-m_{\ell}}\left(2\left(\frac{k_{\ell} m_{\ell}}{\Omega_{\ell}} X_{\ell}\right)^{\frac{1}{2}}\right)}{K_{k_{\ell}-m_{\ell}}\left(2\left(\frac{k_{\ell} m_{\ell}}{\Omega_{\ell}-\Omega_{0, \ell}} X_{\ell}\right)^{\frac{1}{2}}\right)} \\
& \leq \exp \left(2 \sqrt{\frac{k_{\ell} m_{\ell}}{\Omega_{\ell}-\Omega_{0, \ell}} X_{\ell}}-2 \sqrt{\frac{k_{\ell} m_{\ell}}{\Omega_{\ell}} X_{\ell}}\right)\left(\frac{\Omega_{\ell}-\Omega_{0, \ell}}{\Omega_{\ell}}\right)_{\text {(A.2) }}^{\frac{m_{\ell}-k_{\ell}}{2}[32]} \text { We } \\
& \text { Thus, the likelihood ratio can be bounded by } \\
& \quad \mathcal{L}\left(X_{1}, \ldots, X_{L}\right) \leq \prod_{\ell=1}^{L}\left(\frac{\Omega_{\ell}-\Omega_{0, \ell}}{\Omega_{\ell}}\right)^{m_{\ell}} \\
& \quad \times \exp \left(2 \sum_{\ell=1}^{L} \sqrt{k_{\ell} m_{\ell} X_{\ell}}\left[\frac{1}{\sqrt{\Omega_{\ell}-\Omega_{0, \ell}}}-\frac{1}{\sqrt{\Omega_{\ell}}}\right]\right) \text { (A.3) }
\end{aligned}
$$

Replacing the expression of $\Omega_{0, \ell}$ in (14), we get

$$
\begin{aligned}
& \mathcal{L}\left(X_{1}, \ldots, X_{L}\right) \leq \prod_{\ell=1}^{L} \Omega_{\ell}^{-m_{\ell}}\left(\frac{\gamma_{0}}{L}\right)^{\sum_{\ell=1}^{L} m_{\ell}} \\
& \times \exp \left(2 \sqrt{\frac{L}{\gamma_{0}}} \sum_{\ell=1}^{L} \sqrt{k_{\ell} m_{\ell} X_{\ell}}\right) .
\end{aligned}
$$

Let $k_{0}=\max _{1 \leq \ell \leq L} k_{\ell}$ and $m_{0}=\max _{1 \leq \ell \leq L} m_{\ell}$, then we can write

$$
\begin{aligned}
& \mathcal{L}\left(X_{1}, \ldots, X_{L}\right) \leq \prod_{\ell=1}^{L} \Omega_{\ell}^{-m_{\ell}}\left(\frac{\gamma_{0}}{L}\right)^{\sum_{\ell=1}^{L} m_{\ell}} \\
& \times \exp \left(2 \sqrt{\frac{L}{\gamma_{0}}} \sqrt{k_{0} m_{0}} \sum_{\ell=1}^{L} \sqrt{X_{\ell}}\right) .
\end{aligned}
$$

Using Cauchy-Schwarz-Buniakowsky inequality [25, Sec. 11.311], we can write

$$
\sum_{\ell=1}^{L} \sqrt{X_{\ell}} \leq \sqrt{L} \sqrt{\sum_{\ell=1}^{L} X_{\ell}}
$$

Therefore, Eq. (A.5) becomes

$$
\begin{aligned}
& \mathcal{L}\left(X_{1}, \ldots, X_{L}\right) \leq \prod_{\ell=1}^{L} \Omega_{\ell}^{-m_{\ell}}\left(\frac{\gamma_{0}}{L}\right)^{\sum_{\ell=1}^{L} m_{\ell}} \\
& \times \exp \left(2 \frac{L}{\sqrt{\gamma_{0}}} \sqrt{k_{0} m_{0}} \sqrt{\sum_{\ell=1}^{L} X_{\ell}}\right) .
\end{aligned}
$$

Therefore, we obtain the following upper bound

$$
\begin{aligned}
& \mathbb{E}^{*}\left[\mathbb{1}_{\left\{S_{L} \leq \gamma_{0}\right\}} \mathcal{L}^{2}\left(X_{1}, \ldots, X_{L}\right)\right] \leq \prod_{\ell=1}^{L} \Omega_{\ell}^{-2 m_{\ell}}\left(\frac{\gamma_{0}}{L}\right)^{2} \sum_{\ell=1}^{L} m_{\ell} \\
& \times \exp \left(4 L \sqrt{k_{0} m_{0}}\right) .
\end{aligned}
$$

On the other hand, we have that

$$
\bigcap_{\ell=1}^{L}\left\{X_{\ell} \leq \frac{\gamma_{0}}{L}\right\} \subset\left\{\sum_{\ell=1}^{L} X_{\ell} \leq \gamma_{0}\right\} .
$$

This leads to

$$
P \geq \prod_{\ell=1}^{L} \mathbb{P}\left(X_{\ell} \leq \frac{\gamma_{0}}{L}\right) .
$$

We recall that the CDF of a Gamma-Gamma RV is given by

$$
F_{X_{\ell}}(x)=\frac{1}{\Gamma\left(m_{\ell}\right) \Gamma\left(k_{\ell}\right)} G_{1,3}^{2,1}\left[\frac{k_{\ell} m_{\ell}}{\Omega_{\ell}} x \mid \begin{array}{c}
1 \\
k_{\ell}, m_{\ell}, 0
\end{array}\right],
$$

where $G_{p, q}^{m, n}[\cdot]$ is the Meijers G-function defined in [25, Eq. (9.301)]. Around $x=0$, this CDF has the following asymptotic expansion [33, Thm. 1.11]

$$
\begin{gathered}
F_{X_{\ell}}(x) \underset{x \rightarrow 0}{\sim} \frac{\Gamma\left(k_{\ell}-m_{\ell}\right)}{\Gamma\left(k_{\ell}\right) \Gamma\left(m_{\ell}+1\right)}\left(\frac{k_{\ell} m_{\ell}}{\Omega_{\ell}} x\right)^{m_{\ell}}, k_{\ell}>m_{\ell}, \\
k_{\ell}-m_{\ell} \notin \mathbb{N} .
\end{gathered}
$$

A lower bound on $P$ is given by

$$
P \geq \prod_{\ell=1}^{L} F_{X_{\ell}}\left(\frac{\gamma_{0}}{L}\right) \underset{\gamma_{0} \rightarrow 0}{\sim} \prod_{\ell=1}^{L} \frac{\Gamma\left(k_{\ell}-m_{\ell}\right)}{\Gamma\left(k_{\ell}\right) \Gamma\left(m_{\ell}+1\right)}\left(\frac{k_{\ell} m_{\ell} \gamma_{0}}{\Omega_{\ell} L}\right)^{m_{\ell}} .
$$

Thus, we get as $\gamma_{0} \rightarrow 0$

$$
\frac{1}{P^{2}} \leq \prod_{\ell=1}^{L}\left[\frac{\Gamma\left(k_{\ell}\right) \Gamma\left(m_{\ell}+1\right)}{\left(k_{\ell} m_{\ell}\right)^{m_{\ell}} \Gamma\left(k_{\ell}-m_{\ell}\right)}\right]^{2} \Omega_{\ell}^{2 m_{\ell}}\left(\frac{L}{\gamma_{0}}\right)^{2 \sum_{\ell=1}^{L} m_{\ell}} .
$$

Combining (A.8) and (A.14), we obtain

$$
\begin{aligned}
& \limsup _{\gamma_{0} \rightarrow 0} \frac{\mathbb{E}^{*}\left[\mathbb{1}_{\left\{S_{L} \leq \gamma_{0}\right\}} \mathcal{L}^{2}\left(X_{1}, \ldots, X_{L}\right)\right]}{P^{2}} \\
& \leq \prod_{\ell=1}^{L}\left[\frac{\Gamma\left(k_{\ell}\right) \Gamma\left(m_{\ell}+1\right)}{\left(k_{\ell} m_{\ell}\right)^{m_{\ell}} \Gamma\left(k_{\ell}-m_{\ell}\right)}\right]^{2} \exp \left(4 L \sqrt{k_{0} m_{0}}\right) .
\end{aligned}
$$

and hence the proof is concluded.

\section{APPENDIX B \\ PROOF OF THEOREM 2}

To prove Theorem 2, we start by defining the likelihood ratio as

$$
\begin{aligned}
& \mathcal{L}\left(Z_{1}, \ldots, Z_{L}\right)=\prod_{\ell=1}^{L} \frac{f_{Z_{\ell}}\left(Z_{\ell}\right)}{f_{Z_{\ell}}^{*}\left(Z_{\ell}\right)} \\
& =\prod_{\ell=1}^{L}\left(\frac{\Omega_{\ell}-\Omega_{0, \ell}}{\Omega_{\ell}}\right)^{\frac{k_{\ell}+m_{\ell}}{2}} \frac{K_{k_{\ell}-m_{\ell}}\left(2\left(\frac{k_{\ell} m_{\ell}}{\Omega_{\ell}}\right)^{\frac{1}{2}} Z_{\ell}\right)}{K_{k_{\ell}-m_{\ell}}\left(2\left(\frac{k_{\ell} m_{\ell}}{\Omega_{\ell}-\Omega_{0, \ell}}\right)^{\frac{1}{2}} Z_{\ell}\right)} .
\end{aligned}
$$


Using [30, Eq.(1.3)], we can bound the the ratio of the modified Bessel function when $\min _{1 \leq \ell \leq L}\left(k_{\ell}-m_{\ell}\right)>\frac{1}{2}$

$$
\begin{aligned}
& \frac{K_{k_{\ell}-m_{\ell}}\left(2\left(\frac{k_{\ell} m_{\ell}}{\Omega_{\ell}}\right)^{\frac{1}{2}} Z_{\ell}\right)}{K_{k_{\ell}-m_{\ell}}\left(2\left(\frac{k_{\ell} m_{\ell}}{\Omega_{\ell}-\Omega_{0, \ell}}\right)^{\frac{1}{2}} Z_{\ell}\right)} \leq\left(\frac{\Omega_{\ell}-\Omega_{0, \ell}}{\Omega_{\ell}}\right)^{\frac{m_{\ell}-k_{\ell}}{2}} \\
& \times \exp \left(2 \sqrt{k_{\ell} m_{\ell}}\left[\frac{1}{\sqrt{\Omega_{\ell}-\Omega_{0, \ell}}}-\frac{1}{\sqrt{\Omega_{\ell}}}\right] Z_{\ell}\right) .
\end{aligned}
$$

Thereby, we can bound the likelihood ratio by

$$
\begin{aligned}
& \mathcal{L}\left(Z_{1}, \ldots, Z_{L}\right) \\
& \leq \prod_{\ell=1}^{L}\left(\frac{\Omega_{\ell}-\Omega_{0, \ell}}{\Omega_{\ell}}\right)^{m_{\ell}} \exp \left(\sum_{\ell=1}^{L} \frac{2 \sqrt{k_{0} m_{0}}}{\sqrt{\Omega_{\ell}-\Omega_{0, \ell}}} Z_{\ell}\right) .
\end{aligned}
$$

Replacing $\Omega_{0, \ell}$ by its expression (20) and defining $\alpha=$ $\min _{1 \leq \ell \leq L} \alpha_{\ell}$, we obtain

$$
\begin{aligned}
\mathcal{L}\left(Z_{1}, \ldots, Z_{L}\right) & \leq\left(\frac{\eta_{0}}{L}\right)^{2} \sum_{\ell=1}^{L} m_{\ell} \prod_{\ell=1}^{L}\left(\frac{\alpha_{\ell}}{\Omega_{\ell}}\right)^{m_{\ell}} \\
& \times \exp \left(\frac{2}{\eta_{0}} \sqrt{\frac{k_{0} m_{0}}{\alpha}} \sum_{\ell=1}^{L} Z_{\ell}\right) .
\end{aligned}
$$

Therefore, we obtain the following upper bound

$$
\begin{aligned}
\mathbb{E}^{*}\left[\mathbb{1}_{\left\{T_{L} \leq \eta_{0}\right\}} \mathcal{L}^{2}\left(Z_{1}, \ldots, Z_{L}\right)\right] & \leq\left(\frac{\eta_{0}}{L}\right)^{4 \sum_{\ell=1}^{L} m_{\ell}} \prod_{\ell=1}^{L}\left(\frac{\alpha_{\ell}}{\Omega_{\ell}}\right)^{2 m_{\ell}} \\
& \times \exp \left(4 \sqrt{\frac{k_{0} m_{0}}{\alpha}}\right) .
\end{aligned}
$$

A lower bound for the probability $P$ is given by

$$
P \geq \prod_{\ell=1}^{L} F_{Z_{\ell}}\left(\frac{\eta_{0}}{L}\right) .
$$

The square root of Gamma-Gamma RV is given by

$$
F_{Z_{\ell}}(z)=\mathbb{P}\left(Z_{\ell} \leq z\right)=\mathbb{P}\left(X_{\ell} \leq z^{2}\right)=F_{X_{\ell}}\left(z^{2}\right) .
$$

Using (A.12), the expansion of the CDF of $Z_{\ell}$ around $z=0$ is thereby given by

$$
\begin{gathered}
F_{Z_{\ell}}(z) \underset{z \rightarrow 0}{\sim} \frac{\Gamma\left(k_{\ell}-m_{\ell}\right)}{\Gamma\left(k_{\ell}\right) \Gamma\left(m_{\ell}+1\right)}\left(\frac{k_{\ell} m_{\ell}}{\Omega_{\ell}} z^{2}\right)^{m_{\ell}}, k_{\ell}>m_{\ell}, \\
k_{\ell}-m_{\ell} \notin \mathbb{N},
\end{gathered}
$$

Thus, we can write as $\eta_{0} \rightarrow 0$

$$
\frac{1}{P^{2}} \leq \prod_{\ell=1}^{L}\left[\frac{\Gamma\left(k_{\ell}\right) \Gamma\left(m_{\ell}+1\right)}{\Gamma\left(k_{\ell}-m_{\ell}\right)}\right]^{2}\left(\frac{\Omega_{\ell}}{k_{\ell} m_{\ell}}\right)^{2 m_{\ell}}\left(\frac{L}{\eta_{0}}\right)^{4 \sum_{\ell=1}^{L} m_{\ell}} .
$$

Using Eq. (B.5) and (B.9), we get the following upper bound

$$
\begin{aligned}
& \limsup _{\gamma_{0} \rightarrow 0} \frac{\mathbb{E}^{*}\left[\mathbb{1}_{\left\{T_{L} \leq \eta_{0}\right\}} \mathcal{L}^{2}\left(Z_{1}, \ldots, Z_{L}\right)\right]}{P^{2}} \\
& \leq \prod_{\ell=1}^{L}\left[\frac{\Gamma\left(k_{\ell}\right) \Gamma\left(m_{\ell}+1\right)}{\Gamma\left(k_{\ell}-m_{\ell}\right)}\right]^{2}\left(\frac{\alpha_{\ell}}{k_{\ell} m_{\ell}}\right)^{2 m_{\ell}} \exp \left(4 \sqrt{\frac{k_{0} m_{0}}{\alpha}}\right) .
\end{aligned}
$$

which concludes the proof.

\section{REFERENCES}

[1] L. C. Andrews and R. L. Philips, Laser Beam Propagation Through Random Media. Bellingham, WA, USA: SPIE Press, 2005.

[2] A. Abdi and M. Kaveh, "A comparative study of two shadow fading models in ultrawideband and other wireless systems," IEEE Transactions on Wireless Communications, vol. 10, pp. 1428-1434, May 2011.

[3] D. J. Lewinsky, "Nonstationary probabilistic target and clutter scattering models," IEEE Transactions Antenna Propagation, vol. 31, pp. 490-498, May 1983.

[4] M. Gu and D. A. Abraham, "Using McDaniel's model to represent non-Rayleigh reverberation," IEEE Transactions Oceanic Engineering, vol. 26, pp. 348-357, July 2001.

[5] P. M. Shankar, "Error rates in generalized shadowed fading channels," Wireless Personal Communications, vol. 28, pp. 233-238, Feb. 2004.

[6] I. M. Kostic, "Analytical approach to performance analysis for channel subject to shadowing and fading," IEE Proceedings-Communications, vol. 152, pp. 821-827, Dec. 2005.

[7] P. M. Shankar, "Outage probabilities in shadowed fading channels," IEE Proceedings-Communications, vol. 152, pp. 828-832, Dec. 2005.

[8] P. S. Bithas, N. C. Sagias, P. T. Mathiopoulos, G. K. Karagiannidis, and A. A. Rontogiannis, "On the performance analysis of digital communications over generalized-K fading channel," IEEE Commununication Letters, vol. 5, pp. 353-355, May 2006.

[9] L. C. Andrews, R. L. Phillips, and C. Y. Hopen, Laser Beam Scintillation with Applications. Bellingham, Washington: SPIE Press, 2001.

[10] S. Al-Ahmadi, "The Gamma-Gamma signal fading model: a survey," IEEE Antennas and Propagation Magazine, vol. 56, pp. 245-260, Oct. 2014.

[11] M. A. Al-Habash, L. C. Andrews, and R. L. Philips, "Mathematical model for the irradiance probability density function of a laser propagating through turbulent media," Optical Engineering, vol. 40, pp. 1554-1562, Aug. 2001.

[12] P. Deng, M. Kavehrad, Z. Liu, Z. Zhou, and X. Yuan, "Capacity of MIMO free space optical communications using multiple partially coherent beams propagation through non-Kolmogorov strong turbulence," Optics Express, vol. 21, pp. 15 213-15 229, Jul 2013.

[13] N. D. Chatzidiamantis and G. K. Karagiannidis, "On the distribution of the sum of Gamma-Gamma variates and applications in RF and optical wireless communications," IEEE Transactions on Communications, vol. 59, pp. 1298-1308, May 2011.

[14] P. S. Bithas, P. T. Mathiopoulos, and S. A. Kotsopoulos, "Diversity reception over generalized-(KG) fading channels," IEEE Transactions on Wireless Communications, vol. 6, pp. 4238-4243, Dec. 2007. 
[15] E. Bayaki, R. Schober, and R. K. Mallik, "Performance analysis of MIMO free-space optical systems in GammaGamma fading," IEEE Transactions on Communications, vol. 57, pp. 3415-3424, Nov. 2009.

[16] S. Al-Ahmadi and H. Yanikomeroglu, "On the approximation of the generalized-K distribution by a Gamma distribution for modeling composite fading channels," IEEE Transactions On Wireless Communications, vol. 9, pp. 706-713, Feb. 2010.

[17] V. P. Thanh, T. Cong-Thang, and T. P. Anh, "On the MGF-based approximation of the sum of independent Gamma-Gamma random variables," in Proceedings of IEEE 81st Vehicular Technology Conference (VTC Spring), Glasgow, Scotland, May 2015, pp. 1-5.

[18] K. P. Peppas, "A simple, accurate approximation to the sum of Gamma-Gamma variates and applications in MIMO free-space optical systems," IEEE Photonics Technology Letters, vol. 23, pp. 839-841, July 2011.

[19] P. S. Bithas and A. A. Rontogiannis, "Mobile communication systems in the presence of fading/shadowing, noise and interference," IEEE Transactions on Communications, vol. 63, pp. 724-737, Mar. 2015.

[20] J. Morio, M. Balesdent, D. Jacquemart, and C. Vergé, "A survey of rare event simulation methods for static input-output models," Simulation Modelling Practice and Theory, vol. 49, p. 287304, Nov. 2014.

[21] Y. Auffray, P. Barbillon, and J.-M. Marin, "Bounding rare event probabilities in computer experiments," Computational Statistics and Data Analysis, vol. 80, pp. 153-166, 2014.

[22] J. A. Bucklew, Introduction to Rare Event Simulation. New York, NY, USA: Springer, 2004.

[23] N. B. Rached, A. Kammoun, M.-S. Alouini, and R. Tempone, "Unified importance sampling schemes for efficient simulation of outage capacity over generalized fading channels," IEEE Journal of Selected Topics in Signal Processing, vol. 10, pp. 376-388, Mar. 2016.

[24] F. Yilmaz and M.-S. Alouini, "A unified MGF-based capacity analysis of diversity combiners over generalized fading channels," IEEE Transactions on Communications, vol. 60, pp. 862-875, Mar. 2012.

[25] I. S. Gradshteyn and I. M. Ryzhik, Table of Integrals, Series, and Products, seventh edition ed. Amsterdam: Elsevier/Academic Press, 2007.

[26] S. M. Flatté, C. Bracher, and G.-Y. Wang, "Probabilitydensity functions of irradiance for waves in atmospheric turbulence calculated by numerical simulation," Journal of the Optical Society of America, vol. 11, pp. 20802092, July 1994.

[27] Y. Li, M. Piro, and V. Angelakisi, "Design of cellular backhaul topology using the fso technology," in Proceedings of 2nd International Workshop on Optical Wireless Communications (IWOW), Newcastle, UK, Oct 2013, pp. 6-10.

[28] P. J. Smith, M. Shafi, and H. Gao, "Quick simulation: A review of importance sampling techniques in communications systems," IEEE Journal on Selected Areas in Communications, vol. 15, p. 597613, May 1997.
[29] S. Asmussen and P. W. Glynn, Stochastic Simulation : Algorithms and Analysis, ser. Stochastic modelling and applied probability. New York: Springer, 2007.

[30] A. Laforgia and P. Natalini, "Some inequalities for modified Bessel functions," Journal of Inequalities and Applications, vol. 2010, pp. 1-10, 2010.

[31] N. Perlot, Characterization of Signal Fluctuations in Optical Communications with Intensity Modulation and Direct Detection through the Turbulent Atmospheric Channel. Berichte aus der Kommunikationstechnik, Shaker Verlag GmbH, 2005.

[32] T. A. Tsiftsis, "Performance of heterodyne wireless optical communication systems over Gamma-Gamma atmospheric turbulence channels," Electronics Letters, vol. 44, pp. 372-373, Feb. 2008.

[33] A. Kilbas and M. Saigo, H-Transforms : Theory and Applications (Analytical Method and Special Function), 1st ed. Boca Raton, FL, USA: CRC, 2004.

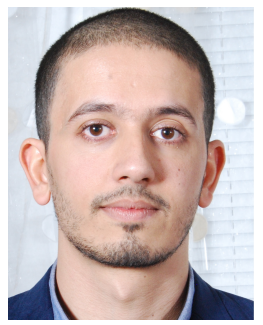

Chaouki Ben Issaid was born in Sfax, Tunisia. He received the Diplôme d'Ingénieur degree from l'École Polytechnique de Tunisie, La Marsa, Tunisia, in 2013. He also holds the Master degree in applied mathematics and computational science from King Abdullah University of Science and Technology (KAUST), Thuwal, Saudi Arabia. Currently, he is working toward the Ph.D degree in statistics at KAUST. His current research interests include efficient Monte Carlo simulations for the performance of wireless communication systems.

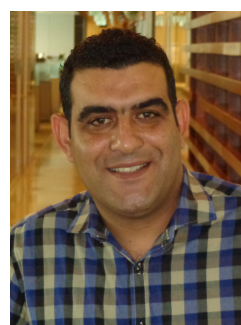

Nadhir Ben Rached was born in Nabeul, Tunisia. He received the Diplôme d'Ingénieur degree from the École Polytechnique de Tunisie, La Marsa, Tunisia, in 2012 and the M.S. degree in Applied Mathematics and Computational Science from King Abdullah University of Science and Technology, Thuwal, Saudi Arabia, in 2013, where he is currently working toward the Ph.D degree in Statistics. His current research interests include rare event simulation algorithms for the accurate performance analysis of wireless communication systems.

Abla Kammoun was born in Sfax, Tunisia. She received the engineering degree in signal and systems from the Tunisia Polytechnic School, La Marsa, and the Master's degree and the Ph.D. degree in digital communications from Télécom Paris Tech [then École Nationale Supérieure des Télécommunications (ENST)]. From June 2010 to April 2012, she has been a Postdoctoral Researcher in the TSI Department, Télécom Paris Tech. Then she has been at Supèlec at the Alcatel-Lucent Chair on Flexible Radio until December 2013. Currently, she is a research scientist at KAUST university. Her research interests include performance analysis, random matrix theory, and semi-blind channel estimation. She is the recipient of the SAM 2014 second Prize Best paper award. 


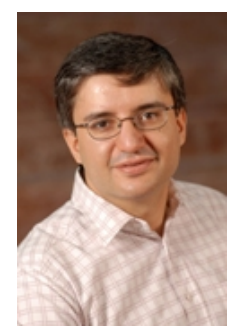

Mohamed-Slim Alouini (S'94, M'98, SM'03, F'09) was born in Tunis, Tunisia. He received the Ph.D. degree in Electrical Engineering from the California Institute of Technology (Caltech), Pasadena, CA, USA, in 1998. He served as a faculty member in the University of Minnesota, Minneapolis, MN, USA, then in the Texas A\&M University at Qatar, Education City, Doha, Qatar before joining King Abdullah University of Science and Technology (KAUST), Thuwal, Makkah Province, Saudi Arabia as a Professor of Electrical Engineering in 2009. His current research interests include the modeling, design, and performance analysis of wireless communication systems.

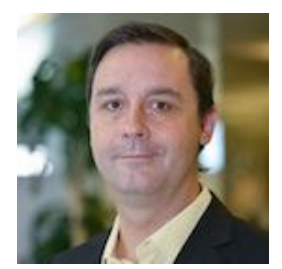

Raul Tempone was born in Montevideo, Uruguay, in 1969. He received the B.E. degree in industrial engineer at the University of the Republic, Montevideo, Uruguay in 1995. After his graduation he worked on the optimal dispatch of electricity for the Uruguayan system using techniques from nonlinear stochastic programming and visited the Royal Institute of Technology (KTH) in Stockholm, Sweden, to study further numerical analysis. He obtained a MSc in Engineering Mathematics in 1999 (inverse problems for incompressible flows, supervised by Jesper Oppelstrup, KTH) and a PhD in Numerical Analysis in 2002 (a posteriori error estimation and control for stochastic differential equations, supervised by Anders Szepessy, KTH). He later moved to ICES, UT Austin, to work as a postdoc from 2003 until 2005 in the area of numerical methods for PDEs with random coefficients (supervised by Ivo Babuska). In 2005 he became an assistant professor with the School of Computational Sciences and the Department of Mathematics at Florida State University, Tallahassee. In 2007 he was awarded the first Dahlquist fellowship by KTH and COMSOL for his contributions to the field of numerical approximation of deterministic and stochastic differential equations. In 2009 he joined KAUST as an Associate Professor in Applied Mathematics (founding faculty). He later became there the Director of the KAUST Center for Uncertainty Quantification and was promoted to Full Professor in 2015.

Dr. Tempone's research interests are in the mathematical foundation of computational science and engineering. More specifically, he has focused on a posteriori error approximation and related adaptive algorithms for numerical solutions of various differential equations, including ordinary differential equations, partial differential equations, and stochastic differential equations. $\mathrm{He}$ is also interested in the development and analysis of efficient numerical methods for uncertainty quantification and Bayesian model validation. The areas of application he considers include, among others, engineering, chemistry, biology, physics as well as social science and computational finance. 